\title{
Molecular cloning and in-silico characterization of high temperature stress responsive $P A P X$ gene isolated from heat tolerant Indian wheat cv. Raj 3765
}

\author{
Jasdeep Chatrath Padaria ${ }^{1 *}$, Harinder Vishwakarma' ${ }^{1}$ Koushik Biswas ${ }^{1}$, Rahul Singh Jasrotia ${ }^{1}$ \\ and Gyanendra Pratap Singh ${ }^{2}$
}

\begin{abstract}
Background: Heat stress leads to accelerated production of reactive oxygen species (ROS) which causes a huge amount of oxidative damage to the cellular components of plants. A large number of heat stress related genes as HSPs, catalases, peroxidases are overexpressed at the time of stress. A potent stress responsive gene peroxisomal ascorbate peroxidase (TapAPX) obtained from heat stress $\left(42^{\circ} \mathrm{C}\right.$ ) responsive subtractive cDNA library from a thermo tolerant wheat CV. Raj3765 at anthesis stage was cloned, characterized and its role was validated under heat stress by proteomics and in-silico studies. In the present study we report the characterization at molecular and in-silico level of peroxisomal TaPAPX gene isolated from heat tolerant wheat cultivar of India.
\end{abstract}

Results: qPCR studies of TapAPX gene displayed up to 203 fold level of expression at $42^{\circ} \mathrm{C}$ heat stress exposure. A full length cDNA of 876 bp obtained by RACE deduced a protein of 292 amino acid residues which gives a complete 3D structure of PAPX by homology modeling. TapAPX cDNA was cloned in expression vector pET28 (a+) and the recombinant protein over-expressed in E. coli BL21 showed highest homology with APX protein as deduced by peptide mass fingerprinting.

Conclusions: TapAPX gene from wheat cV Raj3765 has a distinct role in conferring thermo tolerance to the plants and thus can be used in crop improvement programmes for development of crops tolerant to high temperature.

Keywords: Cloning, In-silico, Peroxisomal ascorbate peroxidase, Homology modeling, Expression, cv. Raj3765

\section{Background}

Heat stress in plants produces large number of Reactive Oxygen Intermediates (ROIs) like superoxide ion $\left(\mathrm{O}^{2-}\right)$, hydroxide ion $\left(\mathrm{OH}^{-}\right)$, singlet oxygen $\left(\mathrm{O}_{2}{ }^{\prime \prime}\right), \mathrm{H}_{2} \mathrm{O}_{2}$ etc. excess of which can lead to damage of plant cells. Among these ROS (Reactive Oxygen Species), $\mathrm{H}_{2} \mathrm{O}_{2}$ can accumulate in cells to toxicity levels because of its high stability. A number of cellular enzymes as superoxide dismutase, monodehydroascorbate reductase, glutathione reductase and ascorbate peroxidase are produced by the cell to get rid of high level of $\mathrm{H}_{2} \mathrm{O}_{2}$. Ascorbate peroxidase plays a leading

\footnotetext{
* Correspondence: jasdeep_kaur64@yahoo.co.in

'Biotechnology and Climate Change Laboratory, National Research Centre on Plant Biotechnology, Pusa Campu, New Delhi 110012, India

Full list of author information is available at the end of the article
}

role in removing ROIs in ascorbate-glutathione cycle [1]. Four types of $A P X$ isoforms have been identified based on the phylogenetic analysis: cytoplasmic $A P X 1$ and $A P X 2$, chloroplastic $A P X$ and membrane bound $A P X$ [2]. Upregulation of $A P X$ genes was observed under abiotic stress conditions in rice, white birch and Suaeda salsa [3-5] and APX has also been reported in different food crops like pea, cayenne pepper, grape [6-8]. $A P X$ thus has a distinct role in conferring tolerance to plants against abiotic stress.

In the present study, the coding sequence of peroxisomal or glyoxisomal Ascorbate peroxidase (TapAPX) gene [Genbank:JX126968] (http://www.ncbi.nlm.nih.gov/) from a heat tolerant cultivar Raj3765 [9] of Indian bread wheat (Triticum aestivum L.) designated as TapAPX was cloned and characterized. The TapAPX gene was subcloned 
in pET-28a and transformed in E. coli for heterologous protein expression studies. The expressed protein TapAPX was confirmed by SDS-PAGE analysis, western blotting and peptide mass fingerprinting. The over expression of TapAPX protein in bacterial system under heat stress was validated and the over-expressed protein was purified using Ni-NTA His-tag purification column for further proteomics studies. Homology search based modeling was performed to deduce a three dimensional (3-D) structure of the protein. The refined structure of generated TapAPX was confirmed with its template structure followed by identification of its active site residues. The functional correlation and interaction between the TapAPX and its substrate $\mathrm{H}_{2} \mathrm{O}_{2}$ was validated by docking analysis.

\section{Results}

\section{Lipid peroxidation assay, subtracted cDNA library} preparation and functional annotation

Estimation of lipid peroxidation was done for the leaf samples collected from plants subjected to heat stress for different time intervals. Non-specific absorbance of the extract at $600 \mathrm{~nm}$ was subtracted from the $532 \mathrm{~nm}$ readings. The MDA (malondialdehyde) concentration in $\mathrm{nmol} / \mathrm{g}$ dry weight (nmol/gDW) was calculated. Samples of heat susceptible cv. HD 2967 subjected to heat stress of $37^{\circ} \mathrm{C}$ and $42^{\circ} \mathrm{C}$ for $30 \mathrm{~min}$ to $6 \mathrm{~h}$, showed statistical significant changes as compared to control (Table 1), an increase in MDA concentration in the range of $40.56 \mathrm{nmol} / \mathrm{gDW}$ to $90.95 \mathrm{nmol} / \mathrm{gDW}$ and $41.99 \mathrm{nmol} / \mathrm{gDW}$ to $108.56 \mathrm{nmol} /$ gDW respectively was observed. Whereas, samples of heat tolerant cv Raj 3765 subjected $37^{\circ} \mathrm{C}$ and $42^{\circ} \mathrm{C}$ heat stress for $30 \mathrm{~min}$ to $6 \mathrm{~h}$ showed increase as well as decrease in MDA concentration in comparison to control. MDA concentration in heat stressed samples of cv Raj 3765 varied from $64.54 \mathrm{nmol} / \mathrm{gDW}$ to $106.67 \mathrm{nmol} / \mathrm{gDW}\left(37^{\circ} \mathrm{C}\right)$ and $44.90 \mathrm{nmol} / \mathrm{gDW}$ to $112.28 \mathrm{nmol} / \mathrm{gDW}\left(42^{\circ} \mathrm{C}\right)$. To identify differentially expressed heat stress responsive genes in wheat cv.Raj 3765 plants at anthesis stage, $42^{\circ} \mathrm{C}$. Heat stress responsive subtractive cDNA libraries were constructed in pGEM-T easy vector. A total of 545 clones were obtained from forward EST (Expressed Sequence Tags) library and colony PCR using T7/SP6 primers confirmed 253 clones to have insert size ranging from 250 to $1500 \mathrm{bp}$. Sequencing of randomly selected 250 clones confirmed a total number of 204 high quality ESTs (http://www.ebi.ac.uk/ena/) after removal of vector (NCBI/vecscreen) and adaptor sequences. After assembly of 204 ESTs [ENA:HG314154HG314357], 149 unigenes containing 45 contigs and 104 singletons were obtained. Similarity analysis of 149 unigene sets by BLASTX search confirmed annotation of 101 unigenes where 48 EST sequences showing no hit (Additional file 1: Table S1).

\section{Real time quantification for TapAPX gene}

Functional annotation of obtained EST sequences identified a number of genes $(5.38 \%)$ expressed in response to abiotic and biotic stress in wheat cv. Raj 3765. A transcript with 720 bp showed highest similarity (97\%) with $A P X$ gene in NCBI database. The differential expression of TapAPX at different stages of wheat development viz seedling, tillering, stem elongation and anthesis stage was observed by qPCR analysis (Figure 1) and fold expression of 203 times of TapAPX at $42^{\circ} \mathrm{C}$ stress during anthesis stage in heat tolerant cv. Raj 3765 was observed. TapAPX was also upregulated at $37^{\circ} \mathrm{C}$ of heat stress during anthesis stage in wheat though the up-regulation was observed to be only 3.2 fold. A base level of gene expression was experienced in heat susceptible wheat $\mathrm{cv}$. HD 2967 during similar stage at heat stress of $37^{\circ} \mathrm{C} \&$ $42^{\circ} \mathrm{C}$. A comparative analysis of expression of TapAPX at other developmental stages (seedling, tillering and stem elongation) in wheat cv. Raj3765 reflected that there was a negative fold change of expression at both $37^{\circ} \mathrm{C}$ and $42^{\circ} \mathrm{C}$ in the above mentioned stages of plant. Housekeeping gene Actin was used as constitutive control for all qPCR studies [10].

\section{Full length characterization of CDNA encoding for TapAPX} gene and its expression in E. coli BL21 cells

Full length cDNA sequence (876 bp) of TapAPX gene was amplified by $5^{\prime}$ and 3' RACE- PCR. The TapAPX cDNA amplicons obtained were cloned in pGEM-T easy vector (Promega, USA) and sequenced to get the full length TapAPX cDNA of 1236 bp. Nucleotide sequence

Table 1 Absolute content of MDA (Malondialdehyde) in $\mathbf{n m o l} / \mathbf{g}$ dry weight showing significant changes

\begin{tabular}{|c|c|c|c|c|}
\hline Time given for heat stress (in $\mathrm{h}$ ) & $\begin{array}{c}\text { HD } 2967,37^{\circ} \mathrm{C} \\
A_{532}-A_{600}\end{array}$ & $\begin{array}{c}\text { Raj } 3765,37^{\circ} \mathrm{C} \text { time } \\
\mathrm{A}_{532}-\mathrm{A}_{600}\end{array}$ & $\begin{array}{c}\text { HD } 2967,42^{\circ} \mathrm{C} \text { time } \\
\mathrm{A}_{532^{-}} \mathrm{A}_{600}\end{array}$ & $\begin{array}{c}\text { Raj } 3765,42^{\circ} \mathrm{C} \text { time } \\
\mathrm{A}_{532^{-}}-\mathrm{A}_{600} \\
\end{array}$ \\
\hline Control & $38.68 \pm 0.13 a$ & $95.56 \pm 0.68 \mathrm{ab}$ & $38.68 \pm 0.13 a$ & $95.56 \pm 0.68 a b$ \\
\hline $1 / 2$ & $90.95 \pm 1.90 b$ & $106.15 \pm 1.11 a$ & $99.86 \pm 2.65 b$ & $44.90 \pm 1.43 d$ \\
\hline 1 & $87.06 \pm 0.47 b c$ & $85.84 \pm 6.17 b$ & $103.91 \pm 3.29 b$ & $112.28 \pm 0.26 a$ \\
\hline 2 & $80.34 \pm 0.42 c$ & $64.54 \pm 0.50 c$ & $108.56 \pm 7.14 b$ & $82.33 \pm 1.64 b$ \\
\hline 4 & $40.56 \pm 2.88 \mathrm{a}$ & $106.67 \pm 1.14 a$ & $69.99 \pm 1.09 c$ & $69.59 \pm 1.15 c$ \\
\hline 6 & $52.82 \pm 0.30 d$ & $64.58 \pm 0.82 c$ & $41.99 \pm 5.51 a$ & $108.17 \pm 7.39 a$ \\
\hline
\end{tabular}

Mean values having the same letter in each column are not significantly different at $P=0.05$ (Tukey test) $(n=3)$. 


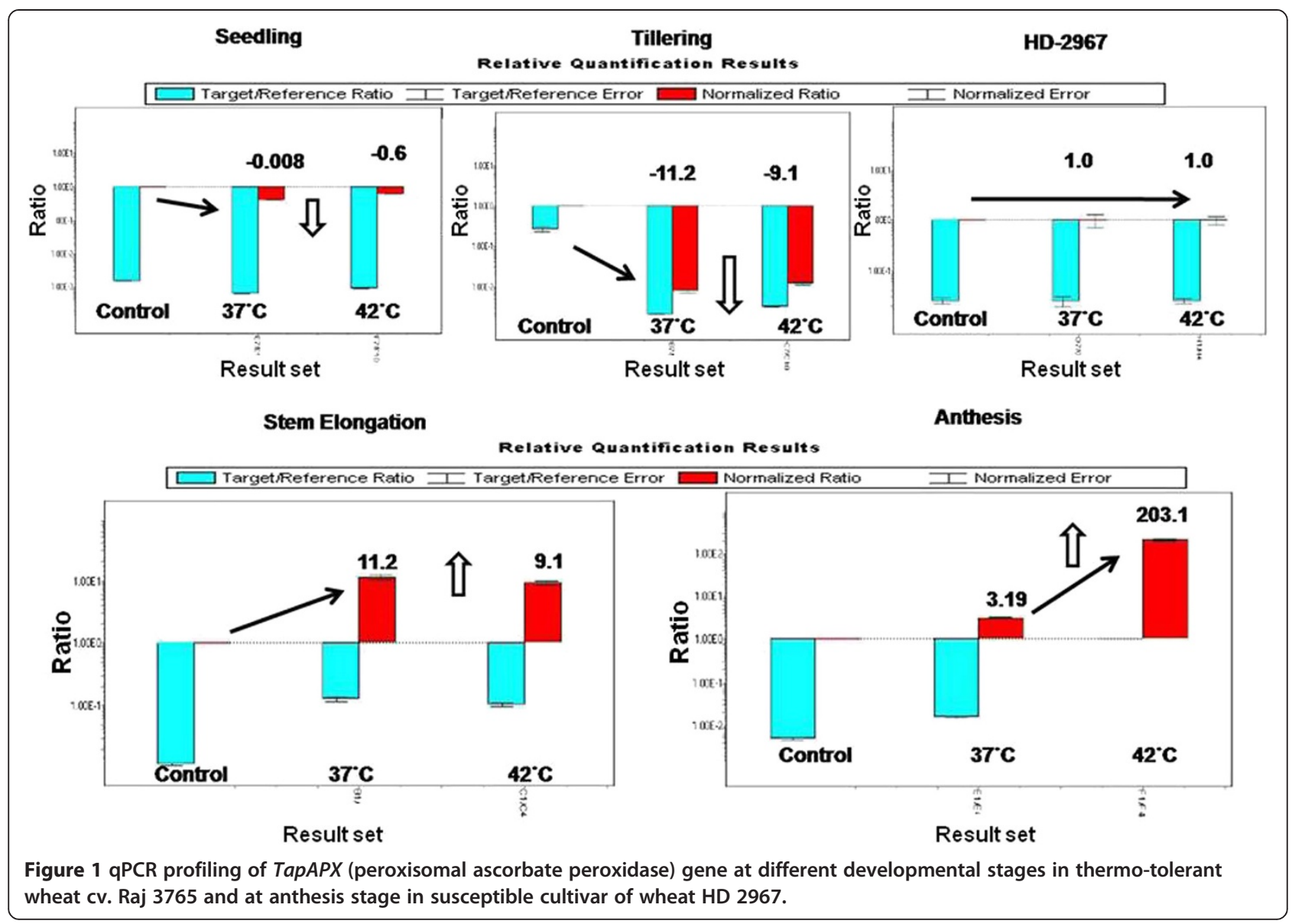

showed 96 percent homology with TapAPX gene in Genbank databases. The obtained TapAPX gene sequence having an ORF of 876 bp with a 199 bp 5' and 161 bp 3' untranslated regions (UTRs) coding a protein of 292 amino acids with a predicted isoelectric point of 7.4 (http://web. expasy.org/translate/). The deduced protein had an approximate molecular weight of $32 \mathrm{kDa}$ and the translated amino acid sequence showed an overall 83 to 98 percent identities with APX from Hordeum vulgare [Genbank:BAB62533], Aegilops tauschii [Genbank:EMT10887], Puccinellia tenuiflora [Genbank:AGW23429], Oryza sativa Japonica [Genbank:NP_001062439], Brachypodium distachyon [Genbank:XP_003574893]. The TapAPX cDNA was cloned in expression vector $\mathrm{pET}-28 \mathrm{a}(+)$ and transformed in $E$. coli BL21. The white colony of E. coli BL21 cells containing pET-28a(+)-TapAPX recombinant plasmid was inoculated in LB media. IPTG was added to the media for induction of $32 \mathrm{kDa}$ fusion protein which was successfully expressed having similar molecular weight of barley $H v A P X$. It was also noticed that the amount of expressed protein was enhanced as the time of IPTG induction increased ( $0 \mathrm{~h}, 3 \mathrm{~h}, 6 \mathrm{~h}$ and $16 \mathrm{~h})$ as evident from the intensity of band on SDS PAGE gel (Figure 2A). This confirmed that the TapAPX protein was expressed in E. coli as in the expected manner. The activity of TapAPX protein expression was detected in bacterial extracts by SDS PAGE showing a prominent and enriched band with an apparent size of $32 \mathrm{kDa}$.

\section{Western blotting, purification and PMF (Peptide mass} fingerprinting) of the expressed TapAPX protein

The expression vector used has His Tag (Histidine Tag) 5' upstream of the cloning site. As a result, the recombinant protein has a $6 \mathrm{X}$ Histidine at $\mathrm{N}$ - terminal. To confirm the expressed recombinant protein, western blotting analysis was carried out with Anti-His antibody for hybridization to His-Tag of recombinant protein. The developed blot showed the presence of a single band of the expected size (Figure 2B). The overexpressed TapAPX protein purified using Ni-NTA column showed the presence of a single band $(\sim 35 \mathrm{kDa})$ (Figure $2 \mathrm{C})$. The sequencing results obtained after PMF of the overexpressed protein band using MALDI-TOF/TOF (Matrix Assisted Laser Desorption/ Ionization-Time of Flight) confirmed the TapAPX protein (Figure 2D). The sequencing results obtained after MALDI showed highest homology with a protein having molecular weight of $31832 \mathrm{Da}$. 

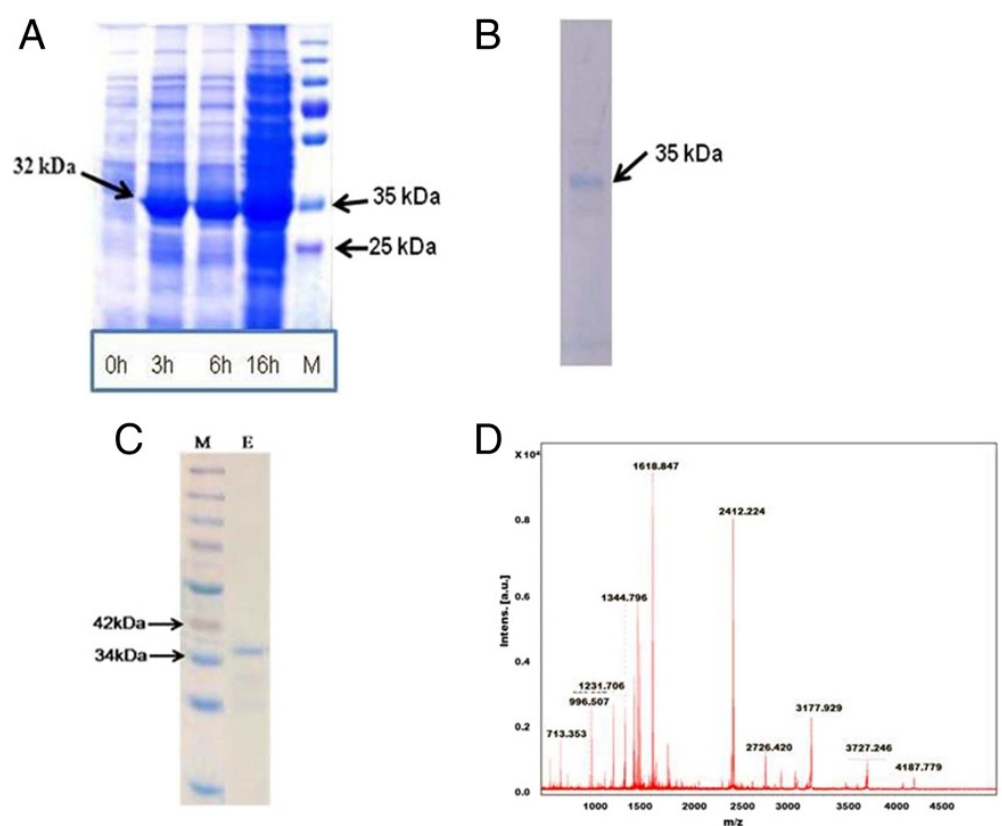

Figure 2 Proteomic analysis of T. aestivum pAPX gene. SDS-PAGE analysis representing the TapAPX protein expression in E. coli BL21 strain grown at different time periods after IPTG induction (A). Western blot analysis of TapAPX protein using Anti-His antibody showing its deduced band of $32 \mathrm{kDa}$ (B). His-tag purification using Ni-NTA column. E- purified recombinant fusion TapAPX protein from E. coli BL21 (pET28a-TapAPX), M-Marker (C). PMF of the over-expressed APX protein using MALDI-TOF/TOF (D)

\section{Heat stress tolerance in $E$. coli}

E. coli cultures harbouring the recombinant plasmid pET-28a-TapAPX grown at temperature viz $37^{\circ} \mathrm{C}, 39^{\circ} \mathrm{C}$, $41^{\circ} \mathrm{C}$ and $43^{\circ} \mathrm{C}$ higher than optimum temperature for $E$. coli growth showed continuous increased growth in comparison to E. coli cells having pET-28a vector only, as evident by O.D. (Optical Density) at A600 of E. coli cultures at different temperatures (Figure 3A, Additional file 2: Table S2). Total protein from bacterial cells of $E$. coli transformed with pET-28a-TapAPX showed over expression of $p A P X$ gene as evident on SDS-PAGE where no expression of TapAPX gene was observed in case of E. coli transformed with pET-28a vector (Figure 3B).

\section{In-silico characterization of TapAPX}

\section{Sequence analysis}

The phylogenetic tree constructed by using full length CDS sequences of Tap $A P X$ gene available in NCBI database

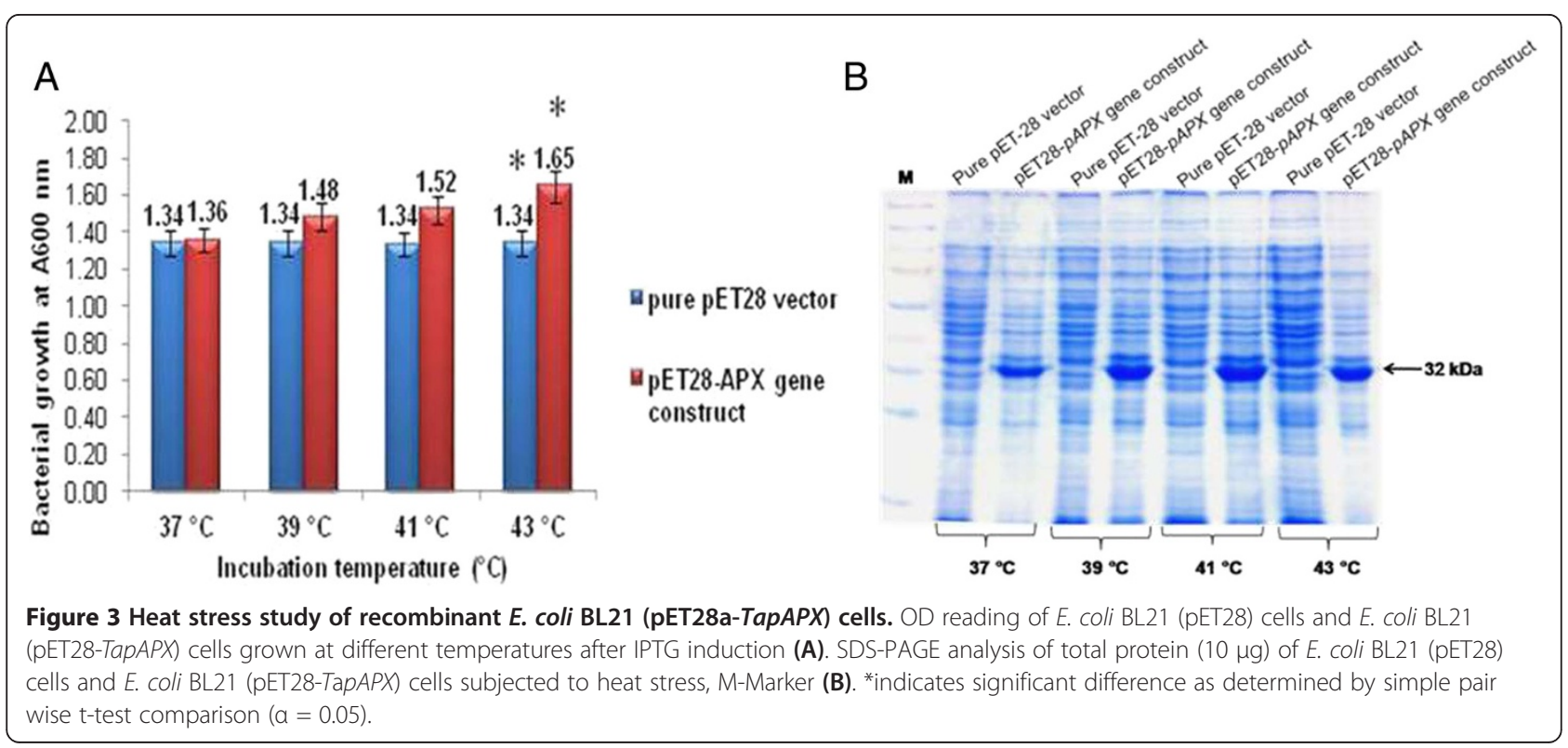


depicts that the present isolate TapAPX well clustered with Triticum aestivum [Genbank:EF555121.1] and Hordeum vulgare [Genbank:AB063117.1] both having 96\% identity whereas only $85 \%$ identity was observed with cluster of Aeluropus littoralis [Genbank:JF907687.1] and Zea mays [Genbank:EU976229.1] (Figure 4A, B) [11]. The protein sequence of TapAPX subjected to PROSITE scan database revealed the presence of 2 functional sites i.e. from residue position number 31-42 and 152-162 and PFAM search database displayed the peroxidase region of TapAPX protein from 15-224. Physiochemical properties of protein obtained from ProtParam tool revealed that the present protein sequence contains 292 amino acids and has a molecular weight of $31770.3 \mathrm{Da}$ with a theoretical pI of 7.74 . Alanine (11.7\%) followed by Leucine (10.3\%) and Glycine (8.6\%) were the maximum number of amino acid residues present in the protein sequence. The total number of negative (Aspartic acid + Glutamic acid) and positively charged (Arginine + Lysine) residues were 39 and 40 respectively. The instability index (II) was computed to be 31.07 and it classifies the protein as stable. The grand average of hydropathicity (GRAVY) was calculated to be -0.270 which indicates the solubility of the protein to be hydrophobic. Secondary structure of TapAPX protein generated by GOR IV method generated an alpha helix region to be of $32.65 \%$, extended strand region of $16.84 \%$ and Random coil region of $50.52 \%$.

\section{Three dimensional structure generation}

The model of wheat TapAPX protein was generated by homology modeling using different servers. The PDB Blast analysis revealed that the protein sequence of TapAPX showed maximum identity (64\%) with Ascorbate Peroxidase of Glycine max [PDB:2XIF_A] (http://www.rcsb.org/ $\mathrm{pdb} /$ home/home.do). On the basis of Ramachandran plot and Verify3D program, the protein structure generated from SWISS-MODEL was selected for further analysis. Structure of TapAPX was visualized using PyMOL (Figure 5A). The PROCHECK analysis of protein revealed that no amino acid residues have phi/psi angles in the disallowed regions (Figure 5B) of Ramachandran plot which indicates that the protein is highly stable. Verify3D program showed good 3D_1D profile score of the residues i.e. $99.17 \%$ residues had an average $3 \mathrm{D}-1 \mathrm{D}$ score of $>0.2$. The QMEAN server used to find the overall quality of three dimensional structure of TapAPX protein displayed a QMEAN and QMEANZ score of 0.816 and 0.5 respectively suggesting that TapAPX protein model is acceptable. The 3D protein model has been submitted to Protein Model Data Base [PMDB:PM0079451] (http://bioinformatics. cineca.it/PMDB/). Comparative study of TapAPX model and its template 2XIF_A by iPBA webserver showed a root mean square deviations (RMSD) score of $0.16 \AA$ as measured by average distance between the backbones of both the models (Figure 5C).

\section{Active site identification and docking study}

Ten different active sites were identified (Figure 6A) in the generated TapAPX $3 \mathrm{D}$ protein model by QSiteFinder (Table 2). $\mathrm{H}_{2} \mathrm{O}_{2}$ ligand molecule was retrieved from pubchem database [PubChem:CID_784] for docking studies of the generated protein structure. Nine different confirmations of docking between the receptor (TapAPX) and the ligand molecule $\left(\mathrm{H}_{2} \mathrm{O}_{2}\right)$ were obtained using
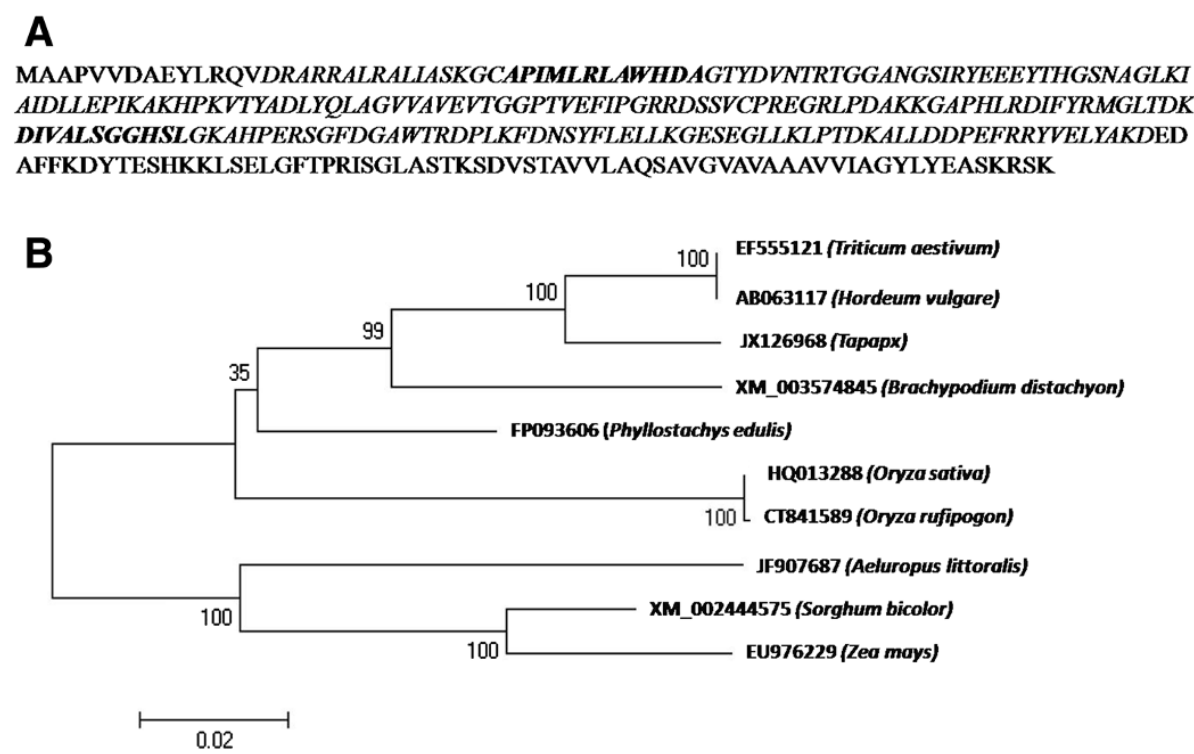

Figure 4 Sequence and phylogenetic analysis. Deduced amino acid sequences of TapAPX gene showing the functional sites domain (bold and italics) and the peroxidase region of TapAPX (italics) (A). Phylogenetic tree analysis of TapAPX gene at nucleotide level from different sources (B). 

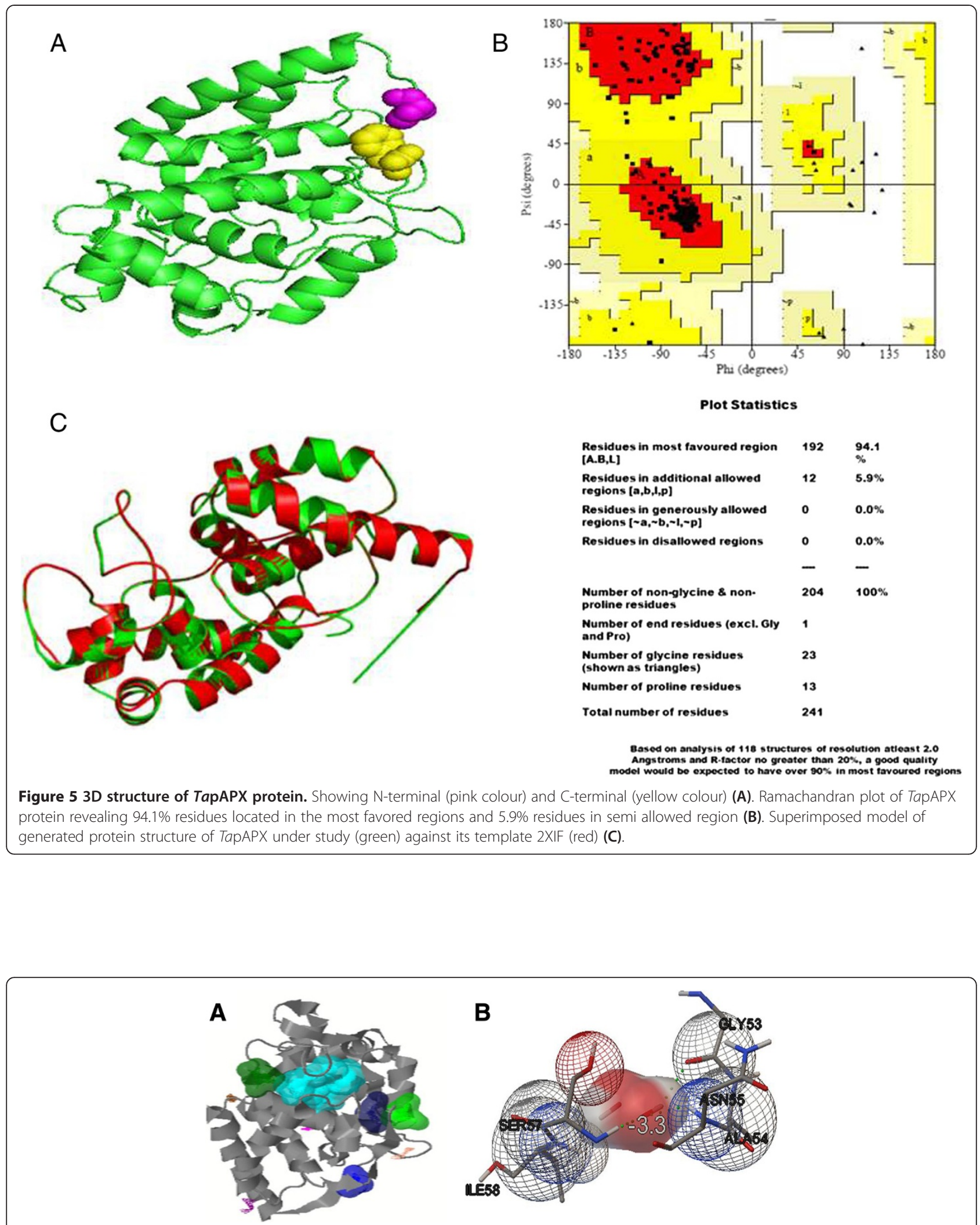

Figure 6 Active sites and interaction of receptor-ligand. Model of generated TapAPX protein showing ten active sites by Q-SiteFinder tool (in different colours) containing different amino acid residues. First five active sites shown in space fill (A). Molecular interaction studies between TapAPX model and substrate $\mathrm{H}_{2} \mathrm{O}_{2}$ by Autodock vina software. Green dots represent the Hydrogen bonding between ASN55 and SER57 (B). 
Table 2 Ten active sites of TapAPX protein model showing its different residues

\begin{tabular}{ll}
\hline Site & Active site residues \\
\hline Site 1 & Lys164, Ala165, His166, Arg169, Ser170, Phe172, Trp176, Tyr187, Leu200, Leu202, Thr204, Asp205, Leu208, Tyr232, His236 \\
Site 2 & Gly43, Thr44, Tyr45, Asp46, Val47, Arg125, Gly127, Arg128, Asp141, Ile142, Phe143, Arg145, Met146 \\
Site 3 & Gly29, Cys30, Ala31, Pro32, Ile33, Leu162, Gly163, Lys164, His166, Arg169, Ala175, Pro180, Leu181 \\
Site 4 & Pro4, Asn55, Gly116, Arg117, Arg118, Ser120 \\
Site 5 & Thr44, His66, Ser68, Asn69, Pro124, Arg125, Glu126, Gly127, Arg128, Leu129, Pro130 \\
Site 6 & Glu9, Tyr10, Arg12, Gln13, Lys85, His86, Pro87, Lys88, Val89 \\
Site 7 & Thr110, Val111, Glu112, Lys230, Thr233, Glu234 \\
Site 8 & Thr51, Gly52, Val122, Cys123, Pro124, Arg125, Arg128 \\
Site 9 & Lys151, Arg216, Tyr217, Leu220, Tyr221, Asp231 \\
Site 10 & Ile25, Gly26, Gly29, Cys30, Ala31, Pro32, Val105, Thr106, Leu181 \\
\hline
\end{tabular}

Autodock vina. The best docked interaction model of TapAPX with $\mathrm{H}_{2} \mathrm{O}_{2}$ (hydrogen peroxide) was analyzed by Autodock tool. Each ligand represents a specific binding energy where the ligand containing lowest binding energy conformation was considered the most acceptable docking structure. The docked confirmation with lowest binding energy score i.e. -3.3 was selected for further analysis. Docking analysis clearly indicates that the ligand molecule was involved in the interaction with TapAPX model and that $\mathrm{H}_{2} \mathrm{O}_{2}$ formed $\mathrm{H}$ bond between 2 amino acid residues i.e. ASN55 and SER57 (Figure 6B).

\section{Discussion}

For cloning of differentially expressed genes, Suppression Subtractive Hybridization (SSH) has proved to be a powerful tool for identifying abiotic stress (heat, drought, salt, nutrient deficiency etc.) responsive gene transcripts in plants $[12,13]$. In our study, the thermo- tolerant wheat cv. Raj 3765 subjected to heat stress of $37^{\circ} \mathrm{C}$ and $42^{\circ} \mathrm{C}$ for different time periods $(1 / 2 \mathrm{~h}, 1 \mathrm{~h}, 2 \mathrm{~h}, 4 \mathrm{~h}$ and $6 \mathrm{~h})$ was selected as tester and normally growing cv. Raj 3765 as control. These plant groups were given heat treatment to get a wide range of heat responsive transcripts expressing at two variable high temperatures. TBARS results with the heat stressed samples showed that MDA concentration increase in case of HD2967 was in a very wide range whereas the heat tolerant cv. Raj 3765 showed MDA variation in a limited range in response to heat stress. Moreover, a rapid decrease in MDA concentration in the heat stress samples of cv. Raj3765 is suggestive of a protection mechanism against oxidative damage due to heat stress which maybe controlled by higher induced activities of antioxidant enzymes $[11,14]$. From the differentially expressed 204 ESTs, which were obtained in subtractive library, $\operatorname{Tap} A P X$ was cloned in full length using RACE-PCR. Heat treatment results in $\mathrm{H}_{2} \mathrm{O}_{2}$ production and $A P X$ plays an important role in eliminating $\mathrm{H}_{2} \mathrm{O}_{2}$ using ascorbate as a specific electron donor. Expression of APX activity was 203 times higher in thermo-tolerant variety as compared to the susceptible one and it was also highly active in $42^{\circ} \mathrm{C}$ rather than $37^{\circ} \mathrm{C}$. The transcript level of TapAPX gene increased gradually at anthesis stage, which is considered as critical developmental stage and is highly sensitive to heat stress [15]. For the qPCR studies, Actin gene was used as internal control, though other housekeeping genes like: Glyceraldehyde-3 phosphate dehydrogenase, 18S rRNA etc. also can be used as internal control in qPCR studies [16]. $A P X$ has been cloned from many other crops like cotton, A. thaliana, barley [17-19] and also from wheat expressing against powdery mildew disease [20]. The role of TapAPX in heat stress response was validated when the gene was expressed in prokaryotic system. Bacterial cells E. coli BL21 harbouring a recombinant plasmid over expressing the TapAPX gene of wheat could tolerate high temperature as evident by a gradual increase of cell density measured by O.D. as compared to cells having pET-28a vector which were sensitive to heat stress.

A heterologous expression system was used for high level expression of TapAPX in E. coli and further facilitated to obtain highly purified TapAPX protein by NiNTA Histidine based purification system. The purified protein could be useful for the production of protein specific antibody. Protein sequence of TapAPX over expressed in bacterial system was confirmed by peptide mass fingerprinting. The molecular interactions of TapAPX with its substrate furnished by computational analysis confirmed its strong connection to degrade ROIs such as $\mathrm{H}_{2} \mathrm{O}_{2}$. The CDS of TapAPX gene could be potentially useful for the development of heat tolerant transgenic crop plants.

The homology search comparative modeling and docking studies finally validated the functional correlation between enzyme TapAPX and its substrate $\mathrm{H}_{2} \mathrm{O}_{2}$. The refined TapAPX 3D structure was successfully generated and its active site residues were identified. 3D structure provides the useful information related to molecular function and identification of active sites [21]. PDB PSIBLAST was searched for finding its template showing 
maximum identity of $64 \%$ that can be considered as a good score to start modeling. It was observed that two distinct amino acid residues viz. Asn and Ser which are potentially involved to recover the normal physiological metabolism against abiotic stress [22,23]. Further detailed molecular biology work on the expression of TapAPX in Arabidopsis plant and common wheat is going on in our laboratory which would provide a valuable work in understanding the mechanism of heat stress tolerance in wheat.

In-silico based approach and characterization of TapAPX at nucleic acid and proteomics level revealed the membrane bound nature of this gene. The nucleotide sequence of TapAPX and its deduced amino acid sequence analysis obtained after PMF (Peptide Mass Fingerprinting) of the differentially expressed protein bands on SDS-PAGE revealed that it belongs to peroxisomal type of peroxidase. In-silico characterization of this gene was carried out by homology BLAST search, multiple sequence alignment, construction of phylogenetic tree, 3D structure and active sites generated by homology modeling and thereby enzyme- substrate interaction study by docking analysis. The docking analysis by Autodock vina tool revealed that hydrogen bonding between $\mathrm{H}_{2} \mathrm{O}_{2}$ with Asn and Ser residues of TapAPX and may cause its breakdown during biochemical reaction. The recombinant TapAPX protein produced in E. coli BL21 cells was able to rescue cells growing at higher temperature $\left(43^{\circ} \mathrm{C}\right)$ as compared to control. The changes in cell growth (in terms of O.D.) in comparison with its control was found to be statistically significant (simple pair wise t-test) when cells were exposed upto $43^{\circ} \mathrm{C}$ stress where it was not changed distinctly for other low temperature stress conditions. In this study, the heat stress was maximized up to $43^{\circ} \mathrm{C}$ for bacterial cells by taking into consideration heat stress imposition at $42^{\circ} \mathrm{C}$ to the plants just before the SSH library construction. However, it is possible that more significant changes may be noticed, if the bacterial cells are exposed to temperature stresses of above $43^{\circ} \mathrm{C}$ and upto a sub lethal temperature. Real time analysis have also shown a very high level gene expression in terms of fold change (F.C.-203) when plants were exposed to heat stress at $42^{\circ} \mathrm{C}$. In vitro results together with in-silico studies confirm the high level of enzyme activity of this gene in order to improve tolerance under abiotic stress and it indicates that the TapAPX gene plays a leading role in mediating overlapping cellular processes especially heat and oxidative stress. This finding will help us to validate not only abiotic stress but also biotic stress response of this enzyme in model plant systems and as well as improvement of genetic background of several crop plants susceptible to abiotic stresses by implying transgenic technologies.

\section{Conclusions}

Complete CDS of TapAPX from thermotolerant wheat cv. Raj3765 was isolated, cloned sequenced and characterized (in-silico) for the first time in Indian bread wheat. qPCR studies confirmed the role of TapAPX gene in thermo tolerance in wheat. The over expressed TapAPX protein was functionally validated in $E$. coli by western blot and MALDI. Biological validation of TapAPX gene in prokaryotic system was confirmed by growth at high temperature of recombinant E. coli cells harbouring wheat TapAPX gene showing significant changes subjected to stress of $43^{\circ} \mathrm{C}$. Ramachandran plot, protein 3D structure and docking analysis have given a deep understanding of TapAPX gene.

\section{Methods}

Plant materials, heat stress treatment, lipid peroxidation assay and SSH cDNA library construction

Heat tolerant wheat (Triticum aestivum) cv. Raj3765 plants and heat susceptible wheat cv. HD2967 [10] plants were grown in National Phytotron Facility, IARI, New Delhi under a light period of $16 \mathrm{~h}$ at $\pm 25^{\circ} \mathrm{C}$ and light intensity of $350 \mu$ molm-2 s-1 and dark period of $8 \mathrm{~h}$ [24]. Heat treatment was given to plants at anthesis stage at $42^{\circ} \mathrm{C}$ for different time periods $(1 / 2 \mathrm{~h}, 1 \mathrm{~h}, 2 \mathrm{~h}$, $4 \mathrm{~h}$ and $6 \mathrm{~h}$ ). Lipid peroxidation assay was performed according to the TBARS (Thiobarbituric Acid Reacting substances) method [25]. Non specific absorbance of the extract at $600 \mathrm{~nm}$ was subtracted from the $532 \mathrm{~nm}$ readings to find out the absolute amount. Total RNA from heat stressed and heat unstressed plants were extracted using Spectrum ${ }^{\mathrm{Tm}}$ Plant Total RNA Kit (Sigma, USA). cDNA was prepared from $1 \mu \mathrm{g}$ of total RNA using SMART PCR cDNA synthesis kit (Clontech laboratories, USA) according to manufacturer's protocol. The forward and reverse libraries were constructed using PCR select cDNA subtractions kit (Clontech laboratories, USA). The expressed secondary PCR amplified products were cloned into pGEM-T easy vector (Promega, USA). The obtained clones of forward and reverse libraries were sequenced in an automated sequencer (ABI Prism 310, Applied Biosystems, USA). All the good EST sequences were assembled into contigs and singlets by using CAP3 sequence assembly program (http://doua.prabi.fr/software/ cap3). The assembled sequences representing unigene data sets were further analyzed for identity search (BLASTX) to the NCBI BLAST program by using BLAST2GO program (www.blast2go.com/b2ghome) for identifying heat stress responsive genes.

\section{Real time PCR of TapAPX transcripts}

Plants at different developmental stages viz. seedling, tillering, stem elongation and anthesis stages were subjected to heat stress treatment in $37^{\circ} \mathrm{C}$ and $42^{\circ} \mathrm{C}$ for different time intervals i.e. $1 / 2 \mathrm{~h}, 1 \mathrm{~h}, 2 \mathrm{~h}, 4 \mathrm{~h}$ and $6 \mathrm{~h}$. Similar heat stress was also imposed to heat susceptible wheat cv. HD2967 at anthesis stage [10] for checking the varietal 


\begin{tabular}{|c|c|c|c|}
\hline S. no. & Gene & Primer sequence & Sequence amplified \\
\hline \multirow[t]{2}{*}{$A$} & Actin & F 5' GAAGCTGCAGGTATCCATGAGACC3' & $151 \mathrm{bp}$ \\
\hline & & R 5' AGGCAGTGATCTCCTTGCTCATC3' & \\
\hline \multirow[t]{2}{*}{ B } & TapAPX & F 5' GATGCTAAGAAAGGCGCACCACAT3' & $124 \mathrm{bp}$ \\
\hline & & R 5' AGGCACATCCTGAAAGGTCTGGTT3' & \\
\hline \multirow[t]{2}{*}{ C } & TapAPX & F 5' CGCGGATCCATGGCGGCTCCGGTGGTGGACG3' & $876 \mathrm{bp}$ \\
\hline & & R 5'CGAGCTCTTACTTGCTCCTCTTGGAAGCCTCGTACAG3' & \\
\hline
\end{tabular}

differences. The stressed and unstressed plant samples were harvested, immediately frozen in liquid $\mathrm{N}_{2}$ and stored at $-80^{\circ} \mathrm{C}$ for downstream experiments. Total RNA was isolated using Spectrum $^{\mathrm{T \mu}}$ Plant Total RNA Kit (Sigma, USA) as per manufacturer's instructions. The cDNA synthesis was carried out from the isolated RNA by using SuperScript $^{\mathrm{TM}}$ III First-Strand Synthesis System (Invitrogen, USA). The qPCR reaction was performed with the synthesized cDNA as template. Based on the sequence information of EST of the forward SSH library, qPCR primers for TapAPX was designed (Table 3). The reaction [Lightcycler 480 SYBR green Master mix, $2 \mathrm{X}-10 \mu \mathrm{l}$ (Roche, USA); PCR primers (Forward and Reverse), $10 \mathrm{mM}-1 \mu \mathrm{l}$ each; cDNA template, $40 \mathrm{ng} / \mu \mathrm{l}-5 \mu \mathrm{l}$ and PCR grade water-3 $\mu \mathrm{l}$ ] was carried out using LightCycler ${ }^{\circledast}$ 480 II System (Roche, USA). For endogenous control, constitutively expressed Actin gene was used. All the reactions were done in triplicate.

\section{RACE (rapid amplification of CDNA ends) PCR of TapAPX} gene and heterologous protein expression in $E$. coli

The 5' and 3' RACE PCR (Rapid amplification of cDNA ends) were performed in separate reactions to obtain full length sequence of TapAPX gene by using SMARTer ${ }^{\text {TM }}$ RACE cDNA Amplification Kit (Clontech laboratories, USA). The fragments obtained after $5^{\prime}$ and $3^{\prime}$ RACEPCR were cloned independently in pGEM-T Easy vector (Promega, USA) and thereafter sequenced to get full length cDNA sequence along with $5^{\prime}$ upstream and 3 ' downstream sequences.

Specific primers (Table 3) were designed for cloning of TapAPX full length cDNA in pET-28a expression vector (Novagen, USA). The oligonucleotide of the primer sequences were designed in a manner to introduce BamHI site just before the start codon ATG and SacI site just after the stop codon (TAA). Using suitable concentration of the designed primers $(10 \mathrm{mM}, 0.5 \mu \mathrm{l}$ each $)$, dNTPs of (25 mM) $0.25 \mu \mathrm{l}, \mathrm{MgCl}_{2}-1.25 \mu \mathrm{l}$, DNA polymerase$0.25 \mu \mathrm{l}$ and DNA polymerase buffer $(10 \times)-2.5 \mu \mathrm{l}$, full length coding TapAPX sequence was PCR amplified using total cDNA (200 ng) as a template. The amplified PCR product was purified using QIAquick PCR purification kit (Qiagen, USA). $1 \mu \mathrm{g}$ of PCR purified product of (TapAPX) was digested with $1 \mu \mathrm{l}$ each of $20 \mathrm{U} / \mu \mathrm{l}$ of restriction enzymes BamH1 and Sac1 (NEB, USA) in a reaction of $20 \mu \mathrm{l}$, the vector pET-28a (500 ng) digested with same set of restriction enzymes. The digestion reaction was carried out at $37^{\circ} \mathrm{C}$ for 3 hours. The digested PCR product was cloned in pET-28a vector using T4 DNA ligase, the ligated product was transformed in $E$. coli DH5 $\alpha$ and recombinant clones were selected on LA plates supplemented with antibiotic Kanamycin $30 \mu \mathrm{g} /$ $\mathrm{ml}$. The positive clones were further screened by colony PCR using gene specific primers of TapAPX. Sequencing of the clone having TapAPX gene was carried out using T7 promoter primer to reconfirm the presence of TapAPX gene along with the presence of $6 \mathrm{X}$ His-tag at the $5^{\prime}$ upstream of the expression vector pET-28a. The expression study of TapAPX gene in prokaryotic system was done by transforming the pET-28a-TapAPX recombinant plasmid in E. coli BL21 cells (Novagen, USA) using heat shock method [26]. The positive clone obtained on selection media ( $\mathrm{LA}+30 \mu \mathrm{g} / \mathrm{ml}$ Kanamycin) was inoculated in LB supplemented with $30 \mu \mathrm{g} / \mathrm{ml}$ kanamycin and incubated at $37^{\circ} \mathrm{C}$. Isopropyl $\beta$-D thiogalacto pyranoside (IPTG), an inducer of $\mathrm{T} 7$ promoter in $\mathrm{pET}-28 \mathrm{a}$ vector, was added at final concentration of $1 \mathrm{mM}$ when O.D of the culture reached an absorbance of 0.5 at $600 \mathrm{~nm}$. TapAPX which is now under the control of $\mathrm{T} 7$ promoter in $\mathrm{pET}-28 \mathrm{a}$ vector, samples were collected at $0,3 \mathrm{~h}, 6 \mathrm{~h}$ and $16 \mathrm{~h}$ after induction was given. The samples were resuspended in protein extraction buffer (100 mM Tris- $\mathrm{HCl}, \mathrm{pH}-7.5,1 \mathrm{mM}$ of PMSF in isopropanol, $10 \mathrm{mM}$ EDTA (Ethylene Diamine Tetra Acetic acid) and $1.6 \mu \mathrm{g} / \mathrm{ml}$ of lysozyme (final concentration) and kept on ice for $1 \mathrm{~h}$. Total protein was quantified using Nanodrop spectrophotometer (Thermo Scientific NanoDrop 2000C Technologies, Wilmington, USA) and $20 \mu \mathrm{g}$ of total protein was loaded on two separate 12\% SDS-PAGE gel [27], one gel was used for coomassie staining to visualize the protein bands and other for western blotting to confirm the identity of protein under study.

Western blotting of TapAPX protein and purification The TapAPX protein from the SDS-PAGE was transferred to the PVDF membrane (BIO-RAD, USA) using 
Mini Trans-Blot ${ }^{\circ}$ cell MTB module (BIO-RAD, USA) using a constant supply of $45 \mathrm{~V}$ for $1 \mathrm{~h}$. The presence of pre-stained marker on the membrane confirmed the transfer process. The membrane was then incubated in blocking solution 5\% BSA (Bovine serum albumin) in TSW buffer (10 mM Tris-HCl, pH-7.4, 0.02\% SDS, 0.9\% $\mathrm{NaCl}, 0.1 \%$ Triton $\mathrm{X}-100$ ) on a gyro-rotary shaker at room temperature for $1 \mathrm{~h}$. Further the membrane was incubated with anti-His-tag antibody (Mouse monoclonal Antibody) (Abm, Canada) at a dilution of 1:4000 for $1 \mathrm{~h}$. Three washes of $10 \mathrm{~min}$ each was given with TSW buffer followed by incubation with Alkaline Phosphatase Conjugated Affinity Purified Antimouse secondary antibody (Abm, Canada) with same dilution and incubated for $1 \mathrm{~h}$. After washing with TSW buffer for 3 times (10 min each), the membrane was developed using NBT/BCIP substrate solution. The presence of single band at appropriate location confirmed the presence of recombinant protein. For protein purification, the total protein was extracted from overnight grown $E$. coli BL21 cell containing pET28a-TapAPX construct by using Total Protein Extraction kit (G-Biosciences, St. Louis, USA). $500 \mu \mathrm{l}$ of the extracted total protein was loaded directly on His SpinTrap columns (GE Healthcare, Amersham, UK) containing Ni Sepharose High Performance medium for perfectly binding of Histidine tagged protein. The purification steps were followed according to manufacturer's protocol and the purified protein was further checked on $12 \%$ SDS-PAGE.

\section{PMF (Peptide mass fingerprinting) of the expressed protein}

The SDS-PAGE gel selected for Coomassie staining having over-expressed TapAPX protein band was sliced out using a sharp scalpel. The gel slice was diced to small pieces and placed in eppendorf tubes. The gel pieces were destained using destaining solution for $10 \mathrm{~min}$ intervals (3-4 times) by vortexing untill the gel pieces become translucent white. The gel pieces were dehydrated using acetonitrile and Speedvac till complete dryness, after that rehydration was done with DTT (Dithiothreitol) and incubated for $1 \mathrm{~h}$. After incubation the DTT solution was removed which was replaced with Iodoacetamide and incubated for $45 \mathrm{~min}$. The supernatant was removed and the gel pieces were incubated with ammonium bicarbonate solution for $10 \mathrm{~min}$. Again supernatant was removed and the gel pieces were dehydrated with acetonitrile for $10 \mathrm{~min}$ and dried using speedvac. Trypsin solution was added to gel pieces and incubated overnight at $37^{\circ} \mathrm{C}$. After incubation the supernatant, which is now having peptides, was transferred to fresh eppendorff tubes. The gel pieces were extracted thrice with extraction buffer and the supernatant was collected each time into the same eppendorff tube and then given Speedvac till complete dryness [28]. The dried pepmix was suspended in TA buffer. The peptides obtained were mixed with ( $\alpha$-cyano-4-hydroxycinnamic acid) HCCA matrix in 1:1 ratio and the resulting $2 \mu \mathrm{lmix}$ was spotted directly onto the MALDI plate. After air drying the sample, it was analyzed on the MALDI TOF/TOF ULTRAFLEX III instrument (Bruker, Germany) and further analysis was done with FLEX ANALYSIS SOFTWARE for obtaining the PEPTIDE MASS FINGERPRINT (PMF). The masses obtained in the peptide mass fingerprint were submitted for Mascot search in "plant" database for identification of the protein.

\section{Heat stress tolerance study in $E$. coli}

The E. coli BL21 cells containing pET28a-TapAPX construct was used for heat stress tolerance study. The initially grown bacterial cell samples at $37^{\circ} \mathrm{C}$ were taken for IPTG induction $(1 \mathrm{mM})$ and thereafter kept at $37^{\circ} \mathrm{C}$, $39^{\circ} \mathrm{C}, 41^{\circ} \mathrm{C}$ and $43^{\circ} \mathrm{C}$ for 6 h. E. coli BL21 cells with pET-28a vector only were used as negative control. The O. D. at $A_{600}$ was measured and the statistical analysis was done using simple pair wise t-test in comparison to respective control at an $\alpha$ level of 0.05 . The total cell protein (10 $\mu \mathrm{g}$ each) from bacterial samples heat stressed at different temperatures was weighed down in each well on a $12 \%$ SDS-PAGE gel to check the expression variation of recombinant protein.

\section{In-silico characterization of TapAPX gene Sequence analysis}

The cloned CDS sequence of TapAPX gene was searched for homology with NCBI database by BLASTN and its translated protein sequence for the complete ORF was retrieved from NCBI database by BLASTP search [29]. The complete sequence of present isolate was compared with reported nine isolates in different monocots available in GenBank. Multiple alignment and sequence identity matrix of the sequences of TapAPX gene was carried out using Clustal Omega program (http://www.ebi.ac.uk/Tools/msa/clustalo/) [30]. Phylogenetic analysis based on neighbor-joining method was conducted in MEGA4 (http://www.megasoftware. net/mega4/mega.html) [31] to investigate the ancestral relationships and closely related species. The protein domain functional analysis of TapAPX protein sequence was searched by PROSITE (http://prosite.expasy.org/) [32], PFAM (http://pfam.xfam.org/) [33] and conserved domain was searched by CDD (http://www.ncbi.nlm.nih. gov/Structure/cdd/cdd.shtml) [34]. The physicochemical properties of TapAPX protein were analyzed by ProtParam tool (http://web.expasy.org/protparam/) [35]. The secondary structure of TapAPX protein was genrated by GOR IV server (http://npsa-pbil.ibcp.fr/cgi-bin/npsa_automat.pl?page=npsa_gor4.html) [36]. 


\section{Three dimensional structure generation}

For the modeling of three dimensional structure, a suitable template was searched by using PDB PSI-BLAST (PositionSpecific Iterated BLAST) [37]. Construction of three dimensional structures by using different homology modeling servers like Phyre2 (http://www.sbg.bio.ic.ac.uk/phyre2/ $\mathrm{html} /$ page.cgi?id=index) [38], ESyPred3D (http://www. unamur.be/sciences/biologie/urbm/bioinfo/esypred/) [39], Protein Structure Prediction Server (PS) ${ }^{2}$ (http://ps2.life.nctu. edu.tw/) [40], SWISS-MODEL (http://swissmodel.expasy. org/interactive) [41], Jigsaw (http://bmm.cancerresearchuk. org/ 3djigsaw/) [42] and I-Tasser (http://zhanglab.ccmb. med.umich.edu/I-TASSER/) [43] was performed to find out the best one. By using SAVES (Structural Analysis and Verification Server) (http://services.mbi.ucla.edu/SAVES/), the conformations of generated models were inspected by the Phi/Psi Ramachandran plot obtained from PROCHECK server [44] and Verify_3D [45] was used to find the acceptable average 3-D ID score. The quality of TapAPX protein models was checked by using Qualitative Model Energy Analysis (QMEAN) server (http://swissmodel.expasy.org/ qmean/cgi/index.cgi) [46]. On the basis of model stability, best model was selected from SWISS-MODEL server. The PyMOL (http://www.pymol.org/) [47] software was used to visualize the 3D structure and the iPBA webserver (http://www.dsimb.inserm.fr/dsimb_tools/ipba/) [48] was used for superimposing the generated model with its template model.

\section{Active site identification and docking study}

The identification of active sites of TapAPX protein structure was obtained from Q-SiteFinder tool (http://www. bioinformatics.leeds.ac.uk/qsitefinder) [49]. Docking was used to identify the specific active sites on protein where receptor- ligand interaction occurs by Autodock vina 1.1.2 (http://vina.scripps.edu/index.html) [50]. The structure of $\mathrm{H}_{2} \mathrm{O}_{2}$ (hydrogen peroxide) ligand molecule available in PubChem site (http://pubchem.ncbi.nlm.nih.gov/) [51] of NCBI database in SDF (Sql Database File) format and the conversion of ligand to PDB format was done using the Open babel software (http://openbabel.org/wiki/Main Page) [52]. The file format conversion of the receptor and ligand structures from PDB to PDBQT was performed by using Autodock tool (ADT) (http://autodock.scripps.edu/ resources/adt) [53]. A grid-box was generated to cover the entire protein structure so that the ligand molecule moves freely. The dimension of grid-box was kept as $22 \AA \times$ $24 \AA \times 28 \AA$ and spacing of grid point set at $1 \AA$.

\section{Availability and requirements}

The software and bioinformatics tools used in this manuscript are mentioned above along with hyperlink.

\section{Additional files}

Additional file 1: Table S1. Representative ESTs of forward $42^{\circ} \mathrm{C}$ heat stress SSH library from wheat. Assembled ESTs displaying a total number of 101 unigenes consisting of 29 contigs (EST. 1-29) and 71 singlets (EST. 30-101).

Additional file 2: Table S2. Table showing the O.D. at $A_{600}$ (in replicate of 4 ) at different temperatures and their average.

\section{Abbreviations}

$\mathrm{H}_{2} \mathrm{O}_{2}$ : Hydrogen peroxide; SDS-PAGE: Sodium dodecyl sulfate polyacrylamide gel electrophoresis; Ni-NTA: Nickel-nitrilotri acetic acid; cv: Cultivar; Da: Dalton; $A_{600}$ : Absorbance at $600 \mathrm{~nm}$; NCBI: National centre for biotechnology information; ENA: European Nucleotide Archive.

\section{Competing interests}

The authors declare that they have no competing interests.

\section{Authors' contributions}

JCP conceived and designed the experiments and contributed in manuscript preparation. HV carried out the cloning of TaPAPX into PET28 vector, western blot analysis and its expression studies in E. coli DH5a and abiotic stress tolerance in E. coli. KB performed CDNA library construction and RACE for full length CDS. RSJ was involved with sequence alignment, phylogenetic analysis, Ramachandran plot, 3D structure generation. GPS has helped in selection and providing of plant material. All authors read and approved the final manuscript.

\section{Acknowledgments}

Authors are thankful to ICAR (Indian Council of Agricultural Research) for providing financial grant under NICRA (National Initiative on Climate Resilient Agriculture) project. Authors are also thankful to the Project Director for providing facilities to carry out research work and Director, IARI for providing facilities for plant stress related experiments at National Phytotron Facility, IARI, New Delhi.

\section{Author details}

${ }^{1}$ Biotechnology and Climate Change Laboratory, National Research Centre on Plant Biotechnology, Pusa Campu, New Delhi 110012, India. ²Division of Genetics, IARI, Pusa Campus, New Delhi 110012, India.

Received: 3 June 2014 Accepted: 2 October 2014

Published: 10 October 2014

\section{References}

1. Asada K: Ascorbate peroxidase hydrogen peroxide scavenging enzyme in plants. Physiol Plant 1992, 85:235-241

2. Teixeira FK, Menezes-Benavente L, Galvao VC, Margis R, Margis-Pinhiero M: Rice peroxidase gene family encodes functionally diverse isoforms localized in different subcellular compartments. Planta 2006, 224:300-314.

3. Lu Z, Liu D, Liu S: Two rice cytosolic ascorbate peroxidase differentially improve salt tolerance in transgenic Arabidopsis. Plant Cell Rep 2008, 26:1909-1917.

4. Wang C, Yang CP, Wang YC: Cloning and expression analysis of an APX gene from Betula platyphylla. J NE Forest U 2009, 37:79-88

5. Ma CL, Wang PP, Cao ZY: CDNA cloning and gene expression of APX in Suaeda salsa in response to salt stress. J Plant Physiol 2002, 28:261-266.

6. Mittler R, Zilinskas BA: Purification and characterization of pea cytosolic ascorbate peroxidase. Plant Physiol 1991, 97:962-968.

7. Yoo TH, Park CJ, Lee GL, Shin RY, Yun JH, Kim KJ, Rhee KH, Paek KH: A hot pepper cDNA encoding ascorbate peroxidase is induced during the incompatible interaction with virus and bacteria. Mol Cell 2002, 14(1):75-84.

8. Lin L, Wang $X$, Wang $Y$ : cDNA clone, fusion expression and purification of novel gene related to ascorbate peroxidase from Chinese wild Vitis pseudoreticulata in E. coli. Mol Biol Rep 2006, 33(3):197-206.

9. Rane J, Pannu RK, Sohu VS, Saini RS, Mishra B, Shoran-Crossa J, Vargas M, Joshi AK: Performance of yield and stability of advanced wheat genotypes under heat stress environments of the Indo-Gangetic plains. Crop Sci 2007, 47:1561-1573. 
10. Padaria JC, Bhatt D, Biswas K, Singh G, Raipuria R: In-silico prediction of an uncharacterized protein generated from heat responsive SSH library in wheat (Triticum aestivum L.). Plant Omics 2013, 6:150-156.

11. DuPont FM, Hurkman WJ, Vensel WH, Tanaka CK, Kothari KM, Chung OK, Altenbach SB: Protein accumulation and composition in wheat grains: effects of mineral nutrients and high temperature. Eur J Agron 2006 25:96-107.

12. Boominathan P, Shukla R, Kumar A, Manna D, Negi D, Verma PK, Chattopadhyay D: Long term transcript accumulation during the development of dehydration adaptation in Cicer arietinum. Plant Physiol 2004, 135:1608-1620.

13. Liu L, Zhou Y, Zhou G, Ye R, Zhao L, Li X, Lin Y: Identification of early senescence- associated genes in rice flag leaves. Plant Mol Biol 2008 67:37-55.

14. Sekman AH, Turkan I, Takio S: Differential responses of antioxidative enzymes and lipid peroxidation to salt stress in salt-tolerant Plantago maritima and salt-sensitive Plantago media. Physiol Plant 2007, 131(3):399-411.

15. Hernandez JA, Almansa MS: Short term effects of salt stress on antioxidant systems and leaf water relations of pea plants. Physiol Plant 2002, 115:251-257

16. Bas A, Fosberg G, Hammarstrom S, Hammarstrom ML: Utility of the housekeeping genes18S rRNA, $\beta$-actin and Glyceraldehyde-3-PhosphateDehydrogenase for Normalization in Real-Time Quantitative Reverse Transcriptase-Polymerase Chain Reaction analysis of Gene Expression in Human T Lymphocytes. Scand J Immunol 2004, 59:566-573.

17. Bunkelmann JR, Trelease RN: Ascorbate peroxidase- a prominent membrane protein in oilseed glyoxysomes. Plant Physiol 1996, 110:589-598.

18. Kubo A, Saji H, Tanaka K, Kondo N: Cloning and sequencing of a cDNA encodingAscorbate peroxidase from Arabidopsis thaliana. Plant Mol Biol 1992, 18(4):691-701.

19. Shi WM, Muramoto $Y$, Ueda A, Takebe T: Cloning of ascobate peroxidase gene from barley and enhanced thermotolerance by overexpressing in Arabidopsis thaliana. Gene 2001, 273(1):23-27.

20. Zhang H, Wang J, Nickel U, Allen RD, Goodman HM: Cloning and expression of an Arabidopsis gene encoding a putative peroxisoma ascorbate peroxidase. Plant Mol Biol 1997, 34:967-971.

21. Katiyar A, Lenka SA, Lakshmi K, Chinnusamy V, Bansal KC: In silico characterization and homology modeling of thylakoid bound ascorbate peroxidase from a drought tolerant wheat cultivar. Genomics Proteomics Bioinformatics 2009, 7:4

22. Kaya C, Aydemir S, Sonmez O, Ashraf M, Dikilitas M: Regulation of growth and some key physiological processes in salt-stressed maize (Zea mays L.) plants by exogenous application of asparagine and glycerol. Acta Bot Croat 2013, 72:157-168.

23. Morimoto Rl: Regulation of the heat shock transcriptional response: cross talk between a family of heat shock factors, molecular chaperones, and negative regulators. Gene Dev 1998, 12:3788-3796.

24. Kumar RR, Sharma SK, Gadpayle KA, Singh K, Sivaranjani R, Goswami S, Rai RD: Mechanism of action of hydrogen peroxide in wheat thermotolerant-interaction between antioxidants isoenzymes, proline and cell membrane. Afr J Biotechnol 2012, 11(78):14368-14379.

25. Heath RL, Packer L: Photoperoxidation in isolated chloroplasts I. Kinetics and stoichiometry of fatty acid peroxidation. Arch Biochem Biophys 1968, 125:189.

26. Bhatt D, Saxena SC, Jain S, Dobriyal AK, Majee M, Arora S: Cloning, expression and functional validation of drought inducible ascorbate peroxidase (Ec-apx1) from Eleusine coracana. Mol Biol Rep 2013, 40(2):1155-1165

27. Sambrook J, Russell DW: Molecular Cloning. In A Laboratory Manual. 3rd edition. New York: Cold Spring Harbor, USA; 2001.

28. Shevchenko A, Tomas H, Havlis J, Olsen JV, Mann M: In-gel digestion for mass spectrometric characterization of proteins and proteomes. Nat Protoc 2006, 1:2856-2860.

29. Altschul SF, Gish W, Miller W, Myers EW, Lipman DJ: Basic local alignment search tool. J Mol Biol 1990, 215:403-410.

30. Slevers F, Wilm A, Dineen D, Gibson TJ, Karpus K, Li W, Lopez R, Macwiliam H, Remmert M, Sodling J, Thompson JD, Higgins DJ: Fast, scalable generation of high quality protein multiple sequence alignments using Clustal Omega. Mol Syst Biol 2011, 7:539.
31. Tamura K, Dudley J, Nei M, Kumar S: MEGA4: Molecular evolutionary genetics analysis (MEGA) software version 4.0. Mol Biol Evol 2007, 24:1596-1599.

32. Sigrist CJA, de Castro E, Cerutti L, Cuche BA, Hulo N, Bridge A, Bougueleret L, Xenarios I: New and continuing developments at PROSITE. Nucleic Acids Res 2012, 41:344-347.

33. Punta M, Coggill PC, Eberhardt RY, Mistry J, Tate J, Boursnell C, Pang N, Forslund K, Ceric G, Clements J, Heger A, Holm L, Sonnhammer ELL, Eddy SR, Bateman A, Finn RD: The Pfam protein families database. Nucleic Acids Res 2012, 40:290-301.

34. Marchler-Bauer A, Zheng C, Chitsaz F, Derbyshire MK, Geer LY, Geer RC, Gonzales NR, Gwadz M, Hurwitz DI, Lanczycki CJ, Lu F, Lu S, Marchler GH, Song JS, Thanki N, Yamashita RA, Zhang D, Bryant SH: CDD: conserved domains and protein three- dimensional structure. Nucleic Acids Res 2013, 41:348-352.

35. Gasteiger E, Hoogland C, Gattiker A, Duvaud S, Wilkins MR, Appel RD, Bairoch A: Protein Identification and Analysis Tools on the ExPASy Server. In The Proteomics Protocols Handbook. Edited by Walker JM. New Jersey: Humana; 2005:571-607.

36. Garnier J, Gibrat JF, Robson B: GOR method for predicting protein secondary structure from amino acid sequence. Methods Enzymol 1996, 266:540-553.

37. Altschul SF, Madden TL, Schäffer AA, Zhang J, Zhang Z, Miller W, Lipman DJ: Gapped BLAST and PSI-BLAST: a new generation of protein database search programs. Nucleic Acids Res 1997, 25:3389-3402

38. Kelley LA, Sternberg MJE: Protein structure prediction on the web: a case study using the Phyre server. Nat Protoc 2009, 4:363-371.

39. Lambert C, Leonard N, De-Bolle X, Depiereux E: ESyPred3D: Prediction of proteins 3D structures. Bioinformatics 2002, 18:1250-1256.

40. Chen CC, Hwang JK, Yang JM: (PS) ${ }^{2}$ : protein structure prediction server. Nucleic Acid Res 2006, 34:152-157.

41. Arnold K, Bordoli L, Kopp J, Schwede T: The SWISS-MODEL Workspace: A web- based environment for protein structure homology modelling. Bioinformatics 2006, 22:195-201

42. Bates PA, Kelley LA, MacCallum RM, Sternberg MJE: Enhancement of protein modelling by human intervention in applying the automatic programs 3D-JIGSAW and 3D-PSSM. Proteins 2001, 45:39-46.

43. Zhang Y: I-TASSER server for protein 3D structure prediction. BMC Bioinformatics 2008, 9:40.

44. Laskoswki RA, MacArthur MW, Moss DS, Thorton JM: PROCHECK: a program to check the stereo chemical quality of protein structures. J Appl Crystallogr 1993, 26:283-291.

45. Luthy $R$, Bowie JU, Eisenberg $D$ : Assessment of protein models with three-dimensional profiles. Nature 1992, 356:83-85.

46. Benkert $P$, Kunzli M, Schwede T: QMEAN server for protein model quality estimation. Nucleic Acids Res 2009, 37:510-514.

47. DeLano WL: The PyMOL Molecular Graphics System. DeLano Scientific California: San Carlos; 2002

48. Gelly JC, Joseph AP, Srinivasan N, de Brevern AG: iPBA: a tool for protein structure comparison using sequence alignment strategies. Nucleic Acids Res 2011, 39:18-23.

49. Laurie AT, Jackson RM: SiteFinder: an energy-based method for the prediction of protein-ligand binding sites. Bioinformatics 2005, 1909:1916

50. Trott O, Olson AJ: AutoDock Vina: improving the speed and accuracy of docking with a new scoring function, efficient optimization and multithreading. J Comput Chem 2010, 31:455-461.

51. Bolton E, Wang Y, Thiessen PA, Bryant SH: PubChem: Integrated Platform of Small Molecules and Biological Activities. In Annual Reports in Computational Chemistry, Volume 4. Washington, DC: American Chemical Society; 2008.

52. O'Boyle NM, Banck M, James CA, Morley C, Vandermeersch T, Hutchison GR: Open Babel: An open chemical toolbox. J Cheminformatics 2011, 3:33.

53. Morris GM, Huey R, Lindstrom W, Sanner MF, Belew RK, Goodsell DS, Olson AJ: Autodock4 and AutoDockTools4: automated docking with selective receptor flexibility. J Comput Chem 2009, 16:2785-2791.

doi:10.1186/1756-0500-7-713

Cite this article as: Padaria et al:: Molecular cloning and in-silico characterization of high temperature stress responsive $P A P X$ gene isolated from heat tolerant Indian wheat cv. Raj 3765. BMC Research Notes 2014 7:713. 